\title{
FOURIER-STIELTJES TRANSFORMS TENDING TO ZERO
}

\section{LAWRENCE J. WALLEN}

Abstract. Let $\mu$ be a Borel measure on the circle, $\hat{\mu}$ its Fourier transform. It is shown that a certain thinness condition on the positive part of the support of $\hat{\mu}$ forces a power of $\mu$ (in the sense of convolution) to be absolutely continuous.

Let $\mu$ be a Borel measure on $[0,2 \pi], \hat{\mu}$ its Fourier transform (i.e. the sequence of Fourier coefficients $\left.\hat{\mu}(n)=\int_{0}^{2 \pi} e^{-i n x} d \mu(x)\right)$, and let $S^{+}$be the set of positive integers $n$ such that $\hat{\mu}(n) \neq 0$. There are many theorems which permit one to conclude that if $S^{+}$is sparse, then $\mu$ is absolutely continuous (this class will henceforth be denoted by A.C.). The classical F. and M. Riesz theorem is, of course, the prototype. It asserts that if $S^{+}$is finite, $\mu \in A$ A.C. Newer and more recondite results permit the same conclusion if $S^{+}$has Hadamard gaps, or $S^{+}$ is the set of perfect squares or $S^{+}$is the set of primes (see [1]).

In this note, we prove an elementary theorem that allows us to infer from a certain thinness condition on $S^{+}$that $\hat{\mu}$ tends to zero. We prove this, not by showing that $\mu \in A$.C., but by showing that some power of $\mu$ in the sense of convolution is in A.C. The thinness condition is similar to one used by Glicksberg to obtain the same sort of conclusion (see [2]). However, Glicksberg's theorem seems to be a harmonic analysis theorem, while our much shallower result seems to be function theoretic.

One last bit of notation is this: $f(\theta) d \theta$ is the measure $\nu$ defined by $d \nu / d \mu=f(\theta)$ for $f \in L^{\infty}$.

THEOREM. Let $S^{+}=\left\{n_{i}\right\}$ be such that $\lim _{i}\left(n_{i+p}-n_{i}\right)=\infty$ for some positive integer $p$. Then

$$
\begin{aligned}
& \mu \star \mu \star \cdots \star \mu \in \text { A.C. } \\
& (p+1) \text { times }
\end{aligned}
$$

Proof. The condition on $\left\{n_{i}\right\}$ is clearly equivalent to the following assertion: given $0<k_{1}<k_{2}<\cdots<k_{p}$, the set of $n$ such that $n \in S^{+}$, $n+k_{1} \in S^{+}, \cdots, n+k_{p} \in S^{+}$is finite. Fixing $k_{1}, \cdots, k_{p}$ for the

Received by the editors July 18, 1969.

AMS Subject Classifications. Primary 4210; Secondary 4225.

Key Words and Phrases. Fourier-Stieltjes coefficients, convolution, absolutely continuous measure, F. and M. Riesz Theorem.

${ }^{1}$ This work was supported by NSF Grant GP 9523. 
moment, we have then that

$$
\hat{\mu}(n) \hat{\mu}\left(n+k_{1}\right) \cdots \hat{\mu}\left(n+k_{p}\right)=\left(\mu \star \exp \left[i k_{1} \theta\right] \mu \star \cdots \star \exp \left[i k_{p} \theta\right] \mu\right) \hat{(}(n)
$$

vanishes for all but a finite set of positive integers $n$. The theorem of F. and M. Riesz implies that $\mu \star \exp \left[i k_{1} \theta\right] \mu \star \cdots \star \exp \left[i k_{p} \theta\right] \mu \in$ A.C. Let $\mu=\alpha+\sigma$ where $\alpha \in A$.C. and $\sigma$ is singular. Since A.C. is an ideal, we have the fact that $\sigma \star \exp \left[i k_{1} \theta\right] \sigma \star \cdots \star \exp \left[i k_{p} \theta\right] \sigma \in \mathrm{A}$.C. Hence if $P(\theta)$ is a trigonometric polynomial of the form $P(\theta)=\sum \alpha_{j} \exp \left[i q_{j} \theta\right]$ where $q_{j}>k_{p-1}$, then $\sigma \star \exp \left[i k_{1} \theta\right] \sigma \star \cdots \star \exp \left[i k_{p-1} \theta\right] \sigma \star P(\theta) \sigma$ $\in$ A.C. According to a famous Theorem of Szegö (see [3]), there exists a sequence $P_{n}$ of such trigonometric polynomials such that $\int\left|1-P_{n}(\theta)\right| d|\sigma| \rightarrow 0$. Thus $p_{n}(\theta) \sigma \rightarrow \sigma$ in the variation norm and so $\sigma \star \exp \left[i k_{1} \theta\right] \sigma \star \cdots \star \exp \left[i k_{p-1} \theta\right] \sigma \star \sigma \in \mathrm{A}$.C. This procedure can now be iterated to finally get $\sigma \star \cdots \star \sigma \in A$.C., concluding the proof.

\section{REFERENCES}

1. Y. Meyer, Spectres des mesures et mesures absolument continues, Studia Math. 30 (1968), 87-99. MR 37 \#3281.

2. I. Glicksberg, Fourier-Stieltjes transforms with small supports, Illinois J. Math. 9 (1965), 418-427. MR 33 \#506.

3. N. I. Ahieser, Lectures on the theory of approximation, OGIZ, Moscow, 1947; English transl., Ungar, New York, 1956. MR 10, 33; MR 20 \#1872.

UNIVERSITY OF HAWAII 